\title{
INTERACTION OF INTERNAL WAVES AND TURBULENCE IN THE OCEAN
}

\author{
A.V. IVANOV, L.A. OSTROVSKY, I.A. SOUSTOVA and L.Sh. TSIMRING \\ Institute of Applied Physics, Academy of Sciences of the U.S.S.R., Uljanov Street 46, \\ 603600 Gorky (U.S.S.R.)
}

(Received July 28, 1982; revised July 1, 1983, accepted July 1, 1983)

\section{ABSTRACT}

Ivanov, A.V., Ostrovsky, L.A., Soustova, I.A. and Tsimring, L.Sh., 1983. Interaction of internal waves and turbulence in the ocean. Dyn. Atmos. Oceans, 7: 221-232.

Within the framework of the semiempirical theory of turbulence for stratified fluids some aspects of the problem of internal wave-turbulence interaction in the upper layer of the ocean are discussed. The conditions of amplification and sustaining of turbulence by internal waves are investigated. Stationary distributions of turbulent energy are found for a stratified fluid with a shear flow produced, for example, by a low-frequency internal wave. The internal wave damping due to both turbulent viscosity and turbulent diffusion in the thermocline is studied. For a two-layer model the damping constant is determined as a function of the wave number. The variation of surface turbulence by internal waves is estimated and the role of this process in slick formation is considered.

\section{INTRODUCTION}

The interaction of internal waves (IW) and small-scale oceanic turbulence is one of the main phenomena determining the formation of the "internal weather" in the ocean. Two of the most important aspects of this problem are the absorption of IW energy by turbulence and turbulence growth and redistribution in space by the IW field. These processes have not yet received due attention, except for some cases of IW breaking producing turbulent spots in a stratified fluid (e.g. Eriksen, 1978), and the influence of turbulent viscosity on IW propagation (Le Blond, 1966).

Two authors (Ostrovsky and Soustova, 1979) made an attempt at a "self-consistent" description of the phenomena using semiempirical equations for turbulent pulsations and variable average motions (IW). Considering IW damping by upper-layer turbulence within the framework of a rather simple model (a two-layer stratification, long IW waves and first mode), they 
proposed a new mechanism of IW damping caused by modulation of turbulent energy by IW in the presence of a buoyancy force.

This paper deals with a wider spectrum of effects of IW-turbulence interaction in the framework of a semiempirical approach to turbulence which may be caused by surface mixing and/or IW breaking. These effects include the growth and redistribution of IW energy in the alternating shear flow, as well as the damping of IW in the near-surface layer. (A generalization from the paper by Ostrovsky and Soustova (1979), which contains an account of multimode propagation and Earth rotation.)

In order to perform a self-consistent analysis of the internal wave-turbulence interaction we use a well-known set of semiempirical equations (Monin and Yaglom, 1971) in a two-dimensional form:

$$
\begin{aligned}
& \mathbf{U}_{\mathrm{i}}+(\mathbf{U} \nabla) \mathbf{U}+(\mathbf{f} \times \mathbf{U})=\frac{\partial}{\partial x_{\mathrm{i}}}\left(K_{\mathrm{i}} \frac{\partial \mathbf{U}}{\partial x_{\mathrm{i}}}\right)-\frac{1}{\rho_{0}} \nabla P+\mathbf{g} \rho / \rho_{0} \\
& \rho_{\mathrm{t}}+(\mathbf{U} \nabla) \rho=\frac{\partial}{\partial x_{\mathrm{i}}}\left(K_{\rho \mathrm{i}} \frac{\partial \rho}{\partial x_{\mathrm{i}}}\right) ; \operatorname{div} \mathbf{U}=0 ; x_{\mathrm{i}}=x, z
\end{aligned}
$$

supplemented by the equations of turbulent energy balance:

$$
\begin{aligned}
B_{\mathrm{t}}+(\mathbf{U} \nabla) B= & \frac{\partial}{\partial x}\left(K_{B x} \frac{\partial B}{\partial x}\right)+\frac{\partial}{\partial z}\left(K_{B z} \frac{\partial B}{\partial z}\right)+K_{z}\left(\frac{\partial U}{\partial z}\right)^{2} \\
& +K_{x}\left(\frac{\partial W}{\partial x}\right)^{2}-K_{\rho z} N^{2}-\epsilon
\end{aligned}
$$

Here, $\mathbf{U}(U, W)$ is the velocity, $P$ is the pressure and $\rho$ is the density averaged over the time interval, $\tau$, which is large compared with the time scale of the turbulence, but small compared with the characteristic IW period; $B=$ $\left\langle U_{\mathrm{i}}^{\prime} U_{\mathrm{i}}^{\prime}\right\rangle$ is the turbulent energy per unit mass or the mean square velocity of turbulent pulsations. Coefficients of turbulent viscosity, $K_{\mathrm{i}}$, are determined, as usual, as the ratio of turbulent Reynolds tension to the averaged (over $\tau$ ) shear of mean flow:

$K_{\mathrm{i}}=-\left\langle U_{\mathrm{i}}^{\prime} U_{\mathrm{j}}^{\prime}\right\rangle / \frac{\partial U_{\mathrm{j}}}{\partial x_{\mathrm{i}}}$

Similarly, the coefficients of turbulent diffusion for density, $K_{\rho \mathrm{i}}$, and turbulent energy, $K_{B \mathrm{i}}$, can be determined:

$K_{\rho \mathrm{i}}=-\left\langle\rho^{\prime} U_{\mathrm{i}}^{\prime}\right\rangle / \frac{\partial \rho}{\partial x_{\mathrm{i}}} ; \quad K_{B \mathrm{i}}=-\left\langle U_{\mathrm{j}}^{\prime} U_{\mathrm{j}}^{\prime} U_{\mathrm{i}}^{\prime}\right\rangle / \frac{\partial B}{\partial x_{\mathrm{i}}}$

where the superscripts denote the turbulent pulsations of corresponding values. The dissipation rate for the kinetic energy of the turbulence is denoted by $\epsilon$, the inertial frequency by $f$, and the Brunt-Väisälä frequency 
by $N$. Further, we shall always use Kolmogorov's closure hypothesis (Monin and Yaglom, 1971), where all coefficients of turbulent diffusion, as well as the rate of turbulent dissipation, are expressed through two variables: turbulent energy and scales of turbulence, $l_{\mathrm{i}}$ :

$K_{\mathrm{i}}=l_{\mathrm{i}} \sqrt{B} ; K_{\rho \mathrm{i}}=a_{\rho} l_{\mathrm{i}} \sqrt{B} ; K_{B \mathrm{i}}=\alpha_{B} l_{\mathrm{i}} \sqrt{B} ; \epsilon=\beta\left(K_{x}^{2} l_{x}^{-2}-K_{z}^{2} l_{z}^{-2}\right) B$

Here, $\alpha_{\rho}, \alpha_{\mathrm{B}}$ and $\beta$ are the empirical constants.

Of course, the semiempirical model used here cannot be considered as a very strict one because it includes closure hypotheses based only on the dimensional analysis. However, models of this type are permanently used in geophysical hydrodynamics (see, for example, Barenblatt, 1982; Fua et al., 1982) and demonstrate a rather good agreement with the experimental data. The most "thin" moment is, in this case, a proper choice of turbulence scales (mixing lengths), $l_{\mathrm{i}}$. Following the hypothesis of Townsend (1976), which states that the small-scale structure of turbulence is determined by the large vortices, one usually accepts that these scales be independent of the coordinates and equal to some fixed parts of the scales of the turbulized region. It means, for example, that in the theory of turbulent erosion of the upper layer the vertical scale of turbulence increases with time (Barenblatt, 1980). In the problems considered here the scales of the turbulized region remain, for all practical purposes, unchanged so it is reasonable to assume the turbulent scales to be constant. There are other closure schemes (see, for example, Monin and Yaglom, 1971), but they lead to similar results after the proper choice of corresponding empirical constants.

\section{AMPLIFICATION AND SUSTAINING OF TURBULENCE}

We begin with the description of turbulent energy variations by a given field of the internal wave governed by eq. 2 . Disregarding the diffusion terms (which is possible if the vertical scale of $\mathrm{IW}, \mathrm{d}_{z}$, is much larger than $l_{z}$ ), a simple equation is obtained:

$B_{\mathrm{t}}=l_{z} B^{1 / 2}\left(U_{z}^{2}+\chi W_{x}^{2}-\alpha{ }_{\rho} N^{2}\right)-\beta B^{3 / 2} l_{z}^{-1}$

where $\chi=l_{z} / l_{x}$ is the empirical constant. As seen from (3), initially weak turbulence increases in a constant horizontal shear flow $\left(W_{x} \equiv 0\right)$ if the Richardson number, $\mathrm{Ri}=N^{2} / U_{z}^{2}$, is smaller than $c_{\rho}^{-1}$ (see also Monin and Yaglom, 1971). Since, in typical cases $c_{\rho} \simeq 0.1$, this condition turns out to be far less rigid than that required for instability in a laminar liquid. The situation is similar though somewhat more complex in an oscillating flow produced by IW. Substituting the values $U\left(x_{\mathrm{i}}, t\right), W\left(x_{\mathrm{i}}, t\right)$ and $N\left(x_{\mathrm{i}}, t\right)$ for a given IW into (3) we obtain the Riccati equation for $B^{1 / 2}$ :

$$
\frac{\partial B^{1 / 2}}{\partial t}=A(x, z, t)-C B-D(z)
$$


where $A(x, z, t)=l_{z}\left(U_{z}^{2}+\chi W_{x}^{2}\right) ; C=\beta l_{z}^{-1}$, and $D(z)=\alpha_{\rho} N^{2} l_{z}$. As an example consider an IW mode in an exponentially stratified fluid (i.e. the non-perturbed value of $N$ is constant). Let

$W(x, z)=W_{0} \sin \left(K_{z} z\right) \cos \left(\omega t-K_{x} x\right)$

and

$U(x, z)=W_{0} K_{z} / K_{x} \times \cos \left(K_{z} z\right) \sin \left(\omega t-K_{x} x\right)$

where $K_{z}$ is determined by the mode number. Then the character of the solution of (3) depends on the wave amplitude parameter, $A_{0}=\frac{1}{2} W_{0}^{2} l_{z}\left(\chi K_{x}^{4}\right.$ $\left.+K_{z}^{4}\right) K_{x}^{-2}$. When $A_{0}<D$ (small-amplitude wave), turbulent energy decays monotonically. If $A_{0}>D$ but $\left(A_{0}-D\right) \ll \omega^{2} / c$ (a moderate-amplitude wave) then the solution of (3) may be found using the averaging method. The turbulent energy may be written in the form $B=B_{0}+B^{\prime}$, where $B_{0}(z, t)$ is the slowly varying part (compared with the IW period) and $B^{\prime} \ll B_{0}$ is the oscillating part. Then, we can easily obtain from (4)

$B_{0}(z, t)=\left[A_{0} \sin ^{2}\left(K_{z} z\right)-D\right] C^{-1} \tanh \left[t / T_{0}(z)\right]$

$B^{\prime}(z, t)=\sqrt{2 A_{0} C} \sin \left(K_{z} z\right) \cdot B_{0}(z, t) / 2 \omega \cdot \cos \left[2\left(\omega t-K_{x} x\right)\right]$

$T(z)=\left\{\left[A_{0} \sin ^{2}\left(K_{z} z\right)-D\right] C\right\}^{-1 / 2}$

It follows from (5) that the turbulent energy is non-zero at $A_{0} \sin ^{2}\left(K_{z} z\right)>D$, i.e. in layers with the maximum amplitude of vertical velocity in an IW mode [between these layers, where $B_{0} \leqslant 0$ in (5), these formulae are not valid and the monotonic decay of $B$ takes place]. The time variation of $B$ at a maximum level is shown in Fig. 1.

For quantitative estimations we take $N=1.1 \times 10^{-2} \mathrm{~s}^{-1}$ and $l_{z}=1 \mathrm{~m}$, and assume empirical constants $\chi$ and $\beta$ to be equal to 100 and 0.05 , respectively (see Barenblatt, 1982). For an IW with $K_{x}=2 \times 10^{-2} \mathrm{~m}^{-1}, \omega=10^{-2} \mathrm{~s}^{-1}$ and $W_{0}=3 \mathrm{~cm} \mathrm{~s}^{-1}$, a characteristic time of turbulence growth $(\tau)$ is $2 \times 10^{3}$ $\mathrm{s}$, while the stationary level of turbulence (asymptotic value of $B_{0}$ ) is $1 \mathrm{~cm}^{2}$ $\mathrm{s}^{-2}$.

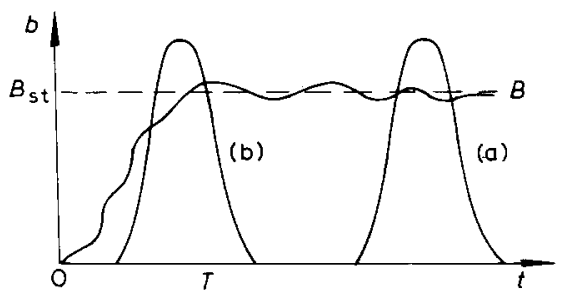

Fig. 1. Turbulent energy amplification due to the oscillating internal wave: (a) low-frequency IW; (b) high-frequency IW, as the characteristic time of turbulence growth. 
For intensive (or low-frequency) IW, when $\left(A_{0}-D\right) \gg \omega^{2} / C$, the effect is considerably different because the oscillations of $B^{\prime}$ are comparable with $B$ itself and the total degeneration of turbulence becomes possible at any period of IW. The solution may now be obtained directly from (4) by neglecting $B_{\mathrm{t}}$ (even if $B_{\mathrm{t}} \neq 0$ in the initial condition, the solution will quickly relax to the one being considered). Then at time intervals where $q=$ $W_{0}^{2} l_{z}\left(\chi K_{x}^{4}+K_{z}^{4}\right) K_{x}^{-2} \sin ^{2}\left(K_{z} z\right) \cos ^{2}\left(\omega t-K_{x} x\right)>D$

$B \simeq(q-D) / C$

and $B=0$ in the opposite case $(q-D<0)$.

If one sets $\omega=2 \times 10^{-3} \mathrm{~s}^{-1}$ and $K_{x}=5 \times 10^{-3} \mathrm{M}^{-1}$ (the remaining parameters are the same as in the previous example) then the peaks of turbulent energy are $B_{\max }=50 \mathrm{~cm}^{2} \mathrm{~s}^{-2}$ and their duration is $-2 \pi / \omega \simeq 3 \times$ $10^{3} \mathrm{~s}$. It is easily seen that the value of $B$ may increase sharply, but then become zero at some moment of each IW period when $\mathrm{Ri}$ approaches $\alpha_{\rho}^{-1}$, i.e. the turbulence (after possible considerable amplification) may be completely suppressed by a strong IW (Fig. 1b). This effect may produce a set of intense local "sparks" of turbulence in the ocean.

It follows from this simple consideration that the hypothesis of sustaining internal oceanic turbulence by IW does not require permanent breaking of the latter. Turbulent spots produced by occasional breakings (where $\mathrm{Ri}<$ 1/4) may be preserved from degeneration by much weaker IW $\left(\mathrm{Ri}<a_{\rho}^{-1} \simeq\right.$ 10). This possibility seems to be an important modification of the usual approach to the problem of the genesis of small-scale turbulence in the ocean.

For yet longer or low-frequency waves, diffusion should be taken into account. The non-stationary problem is rather complicated; however, stationary solutions of (1) and (2) give some information on the role of diffusion effects, considering IW as a shear flow, $U_{0}(z)$, without horizontal variations. After double integration of (1) and (2) we have:

$\left(\frac{2}{3} \cdot \alpha_{\rho} / l_{z}^{2}\right) \frac{\mathrm{d}^{2} R}{\mathrm{~d} z^{2}}+C_{1}^{2} R^{-1 / 3}-\alpha_{\rho} g / \rho_{0} C_{2}-\beta R / l_{z}^{4}=0$

$R^{1 / 3} \frac{\mathrm{d} U_{0}}{\mathrm{~d} z}=C_{1}, R^{1 / 3} \frac{\mathrm{d} \rho_{0}}{\mathrm{~d} z}=C_{2}$

where $R=\left(l_{z} \sqrt{B}\right)^{3}$, and $C_{1,2}=$ constant. Figure 2 shows the phase trajectories of eq. 7 and the respective vertical variations of the turbulent energy, $B_{0}(z)$, and density, $\rho_{0}(z)$. The character of the solution depends essentially on the presence of the mean flow. If $C_{1}=0$ (Fig. 2a) limited distributions $B_{0}(z)$ and $\rho_{0}(z)$ occur only, due to the non-zero turbulent energy flux across the oceanic surface $(z=0)$. This case corresponds to a well-known model of the upper layer in which turbulence vanishes at a finite depth (Kitaigorodsky and Miropolsky, 1967). 
If, however, $C_{1} \neq 0$ (a shear flow is present), localized solutions describing turbulized layers with sharp boundaries also exist (Fig. 2b). The thickness, $d$, of these layers depends on the turbulent energy density, $B$, per unit area of
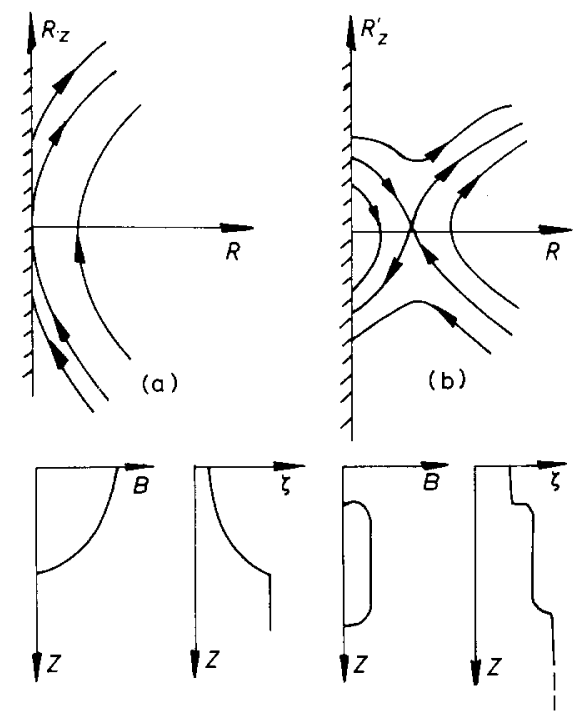

Fig. 2. Phase portraits of eqn. 5 and the corresponding density and turbulent energy profiles for $C_{1}=0$ (a) and $C_{1} \neq 0(\mathrm{~b})$.

the layer:

$d \simeq \beta / \propto_{\rho} B\left[N_{0}^{4} l_{z}^{2}\left(1-\alpha_{\rho} \operatorname{Ri}_{0}\right)\right]^{-1}$

where $N_{0}$ and $\mathrm{Ri}_{0}$ are the Brunt-Väisälä frequency and the Richardson number, respectively, in the external flow. At $\mathrm{Ri}_{0} \rightarrow \alpha_{\rho}^{-1}$ and a given vertical size of the layer the turbulent energy density in the spot tends to zero. At $N_{0}=3 \times 10^{-2} \mathrm{~s}^{-1}, \mathrm{Ri}_{0}=8, l_{z}=1 \mathrm{~m}$, and $d=2.5 \mathrm{~m}$ we have $B \simeq 100 \mathrm{erg} / \mathrm{g}$ $\mathrm{cm}^{2}$. The fact that stationary layers may occur in a locally constant shear flow of a stratified fluid may be of interest in connection with the problem of oceanic fine structure.

\section{INTERNAL WAVE DAMPING}

Now consider the problem of weak IW propagation in the presence of a stationary distribution of turbulent energy. Since the IW perturbs stationary profiles $B_{0}(z)$ and $\rho_{0}(z)$, the solution of the system (1) and (2) has the form: $\rho=\rho_{0}(z)+\rho^{\prime}, B=B_{0}(z)+B^{\prime}, \mathbf{U}=\mathbf{U}^{\prime}$

etc. The superscripts denote perturbations produced by the IW which are 
supposed to be proportional to $\exp \left[i\left(\omega t-K_{x} x\right)\right]$. After linearization of eqs. 1 and 2, and simple transformations we obtain an equation for the amplitude of the vertical velocity component in the IW:

$$
\begin{aligned}
\frac{\mathrm{d}^{2} W^{\prime}}{\mathrm{d} z^{2}}+K_{x}^{2}\left(\frac{N^{2}-\omega^{2}}{\omega^{2}-f^{2}}\right) W^{\prime}= & (i \omega)^{-1}\left\{\frac{\mathrm{d}^{2}}{\mathrm{~d} z^{2}}\left(K_{z}^{0} \frac{\mathrm{d}^{2} W}{\mathrm{~d} z^{2}}\right)\right. \\
& -\frac{K_{x}^{2} \omega^{2}}{\omega^{2}-f^{2}} \frac{\mathrm{d}}{\mathrm{d} z}\left[\left(K_{x}^{0}+K_{z}^{0}\right) \frac{\mathrm{d} W^{\prime}}{\mathrm{d} z}\right] \\
& \left.-\frac{K_{x}^{2}}{\omega^{2}-f^{2}} \frac{\mathrm{d}^{2} K_{\rho z}^{0}}{\mathrm{~d} z^{2}}\left(N^{2} W^{1}\right)\right\}
\end{aligned}
$$

Next, assume that IW damping is small at the wavelength scale. In this case the solution of (9) may be obtained using the perturbation theory. Let $W^{\prime}=W_{0}+W^{\prime}$ and $K_{x}=K_{0}+K_{1}+\ldots$, where $K_{0}$ and $W_{0}$ satisfy eq. 9 without the right-hand side. The condition of finiteness of $W^{\prime}$ leads to an equation for $K_{1}$, with the imaginary part of $K_{1}$ defining the IW damping (cf. Ostrovsky and Soustova, 1979). Such a scheme may be constructed for arbitrary stratification defined by stationary eq. 7. As has been mentioned this problem was considered in an earlier paper (Ostrovsky and Soustova, 1979) for a specific model of a two-layer non-rotating fluid. Here, we take into account stratification of the lower layer assuming that:

$N(z)=\begin{aligned} & N_{1}(0>z>-h) \\ & N_{2}(-h>z>-H)\end{aligned}$

together with density discontinuity, $\Delta \rho$, at $z=-h$. This permits us to consider a multimode situation. The Koriolis effects, which were not considered before, turn out to be important for the low-frequency part of the IW spectrum.

Assume that turbulence is distributed in accordance with eq. 7 at $U \equiv 0$ (Fig. 2a), i.e. decays with increasing depth from the maximum value near the surface to zero at $z=-h$, and is absent under the thermocline $(z<-h)$. Thus, the IW energy is transformed not only into kinetic energy of turbulence (and eventually into heat losses) as in homogeneous fluids, but also into potential energy (through an additional turbulent mixing associated with the perturbation of turbulent energy). Then eq. 9, with the right-hand side equal to zero is valid for $z<-h$, while eq. 7 is satisfied by the equilibrium state with $C_{1}=0$ and the given fluxes of buoyancy and turbulent energy across the free surface. Usual approximations of the rigid surface and bottom, together with the condition of the pressure and normal velocity continuity at $z=-h$ taken as boundary conditions yield: 
$W_{1}=W_{2}(-H)=0 ; \quad W_{1}(-h)=W_{2}(-h)$

$\left(\frac{\mathrm{d} W_{1}}{\mathrm{~d} z}-\frac{\mathrm{d} W_{2}}{\mathrm{~d} z}-\frac{g K_{x}^{2}}{\omega^{2}} \frac{\Delta \rho}{\rho} W_{1}\right)_{z=-h}=0$

where 1 and 2 correspond to the regions above and below the thermocline. Omitting all mathematical operations (see Ostrovsky and Soustova, 1979 for a simpler case) we can give a final expression for the damping constant, Im $\omega$, in the range where waves are long compared to the depth of the layer:

$\operatorname{Im} \omega=V_{\mathrm{gr}} / V_{\mathrm{ph}}\left(\frac{N_{2} H}{n \pi}\right)^{2}\left\{\frac{K_{0}^{2} K_{x}(0)}{(\Delta \rho / \rho) g h}+\frac{K_{\rho z}(0) N_{1}^{2} K_{0}^{2}}{(\Delta \rho / \rho) g l_{z}\left[f^{2}+K_{0}^{2}\left(\frac{N_{2} H}{n \pi}\right)^{2}\right]}\right\}$

where $V_{\mathrm{ph}}$ and $V_{\mathrm{gr}}$ are the phase and group velocities, respectively, of the mode, and $n$ is the IW mode number. The first term of the right-hand side of (10) represents the effect of turbulent viscosity in the upper layer, while the second term is responsible for the turbulent diffusion effects being proportional to the buoyancy flux across the ocean surface, $C_{2}=K_{\rho z}(0) N_{1}^{2}$. It is easy to see that for low-frequency waves $\left(\omega \ll N_{2}\right)$ the contribution of the latter term dominates. Figure 3 shows $\operatorname{Im} \omega$ as a function of $K_{0}$. In Fig. 4 the energy dissipation rate, $\dot{E}_{n}\left(K_{x}\right)$, for the Garrett-Munk spectrum (Garrett and Munk, 1975) is given. For comparison a "viscous" part of the decrement is shown in Fig. 4. It is seen that in the "energy-carrying" range the buoyancy effect prevails.

Using these results one can make an integral estimation of characteristic

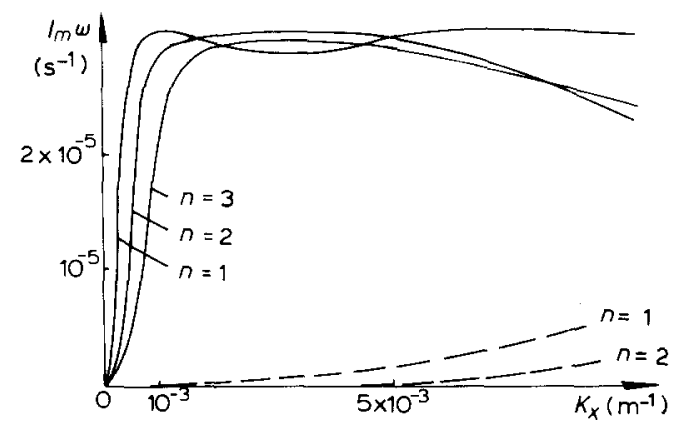

Fig. 3. IW damping constant as a function of the wave number for the first three modes, at $h=50 \mathrm{~m}, H=10^{3} \mathrm{~m}, \Delta \rho / \rho=10^{-3}, N_{1}=5 \times 10^{-4} \mathrm{~s}^{-1}, N_{2}=3 \times 10^{-5} \mathrm{~s}^{-1}$ and $K_{z}(0)=3 \times 10^{-4}$ $\mathrm{m}^{2} \mathrm{~s}^{-1}$. A dashed line shows the contribution of a conventional turbulent viscosity [the first term in (10)]. 


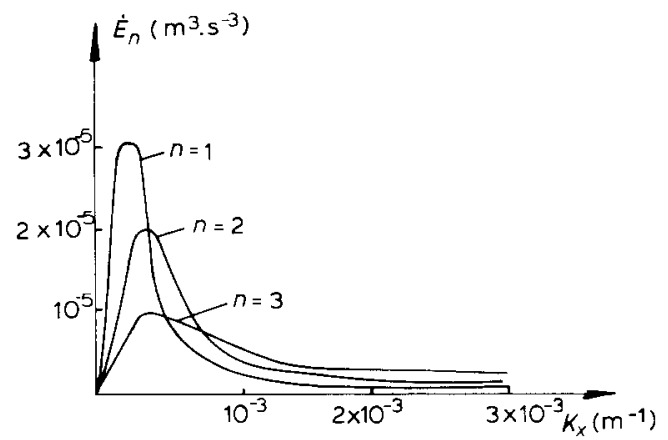

Fig. 4. Energy dissipation rate as a function of the wave number for the Garrett-Munk spectrum (the parameters are the same as in Fig. 3).

dissipation time for the total IW energy:

$\tau=E / \sum_{n=1}^{6} \int_{0}^{\infty} \dot{E}_{n}(K) \mathrm{d} K \sim 20$ days

where $E$ is the energy density in the Garrett-Munk (1975) spectrum.

The process of IW damping due to upper-layer turbulence is, naturally, accompanied by changes in turbulent energy that lead to changes of mean density stratification. The existence of averaged (or more exactly, slowly varying compared to the wave period) deformation of mean profiles in the thermocline region follows from the non-linearity of our basic equations ( 1 and 2). Using the perturbation method one can find the small (of the square order over IW amplitude) deviations of undisturbed profiles of density and turbulent energy. As follows from the analysis (Ostrovsky et al., 1981) the IW mode causes intensification of turbulence and distortion of the density gradient in the pycnocline. The characteristic time of these variations is of the order of the IW damping time. If IW is a train of finite length, then after passing the train these variations disappear for the characteristic diffusion time, which may achieve a few hours.

\section{MODULATION OF SURFACE TURBULENCE BY INTERNAL WAVES AND SLICK FORMATION}

Finally, let us discuss the modulation of near-surface turbulence by IW as a mechanism for the IW manifestation on the ocean surface. This effect leads to variation of the damping constant of the surface waves, and as a result to the modulation of the intensity of the surface waves.

The formulation of the problem is the same as in Section 3. A long IW propagates along the thermocline, the upper layer being turbulized. The 
oscillating perturbations of turbulent energy may be found from the linearized approach developed in Section 3 (see eqs. 8 and 9). We now consider the region just near the ocean surface where the values of $N_{0}$ and $B_{0}$ are partially dependent on depth. In this case we have (Pelinovsky and Soustova, 1980):

$\frac{\mathrm{d}^{2} B^{\prime}}{\mathrm{d} z^{2}}-A B^{\prime}=\frac{a_{\rho}}{a_{B}} \frac{g}{\rho_{0}} \frac{\mathrm{d} \rho^{\prime}}{\mathrm{d} z}$

where

$A=\frac{i \omega}{\alpha_{\rho}} K_{z}^{0}+\frac{1}{2} \frac{\alpha_{\rho}}{\alpha_{B}} \frac{N_{0}^{2}}{B_{0}}-\frac{3}{2} \frac{\beta}{\alpha_{B} l_{z}^{2}}+\frac{3}{2} \chi K_{x}^{2}$

If the intensity of the background turbulence near the surface is not very large $\left(K_{z}^{0} K_{z}^{2} \ll \omega\right)$, then in (11) $\mathrm{d}^{2} B^{\prime} / \mathrm{d} z^{2} \ll A B^{\prime}$, and $B^{\prime}$ is expressed as follows:

$B^{\prime}=-\frac{a_{\rho}}{a_{B}} \frac{g}{\rho_{0} A} \frac{\mathrm{d} \rho^{\prime}}{\mathrm{d} z} \simeq-i \sqrt{\mathrm{Ri}_{\tau}} \alpha_{\rho} N_{0} \omega^{-1} B_{0}\left(U^{\prime} / V_{\mathrm{ph}}\right)$

where $V_{\mathrm{ph}}=\omega / K_{x}$ is the phase velocity of the internal wave and $\mathrm{Ri}_{\tau}=$ $l_{z}^{2} N_{0}^{2} / B_{0}$ is the turbulent Richardson number. As an estimation let us take $\omega=3 N_{0}$ (but, of course, $\omega<N_{\max }$, where $N_{\max }$ is a maximum value of $N$ ) and $\mathrm{Ri}_{\tau} \simeq 1$, then $\left|B^{\prime} / B_{0}\right| \simeq 0.1 U / V_{\mathrm{ph}}$. It can readily be seen that $\left|B^{\prime} / B_{0}\right|$ $B_{0}^{-1 / 2}$, so this ratio increases as the unperturbed turbulent pulsations become weaker (of course, the semiempirical hypothesis should be valid for such values of $B_{0}$ ).

Modulation of surface turbulence by IW may result in the modulation of the damping constant of surface waves and, hence, in the formation of slicks (Phillips, 1977). Consider this effect on the basis of a simple linear model of surface roughness (Pelinovsky, 1978) valid for a weak wind. The model assumes that the surface waves are produced by pulsations of atmospheric pressure and the stationary state may only be established due to viscous dissipation.

In order to estimate the surface roughness variation we use the equation for the surface wave amplitude, $a\left(K_{\mathrm{s}}\right)$, in the form (Pelinovsky, 1978):

$\frac{\partial}{\partial t}\left\langle a^{2}\left(K_{\mathrm{s}}\right)\right\rangle=F\left(K_{\mathrm{s}}\right)-2 K_{x}(x, t) K_{\mathrm{s}}^{2}\left\langle a^{2}\left(K_{\mathrm{s}}\right)\right\rangle$

where $K_{\mathrm{s}}$ is the wave number of the surface wave, $F\left(K_{\mathrm{s}}\right)$ describes the forcing associated with atmospheric pulsations, and $K_{x}$ is the turbulent viscosity. For a quasi-stationary process (when the surface roughness follows the variation of $K_{x}$ ) one obtains

$\left\langle a^{2}\left(K_{\mathrm{s}}\right)\right\rangle=F\left(K_{\mathrm{s}}\right) / 2 K_{x} K_{\mathrm{s}}^{2} \simeq \frac{F\left(K_{\mathrm{s}}\right)}{2 K_{x}^{0}}\left(1-\frac{1}{2}\left|B^{\prime} / B_{0}\right|\right)$ 
Thus, the surface constant (the amplitude ratio of the surface waves in the maximum and in the minimum) is of the order of $B^{\prime} / B_{0}$, and may amount to several decibels for sufficiently intense IW. This mechanism of slick formation may be notable in a real ocean together with the known mechanisms of surface wave transform in inhomogeneous flows of water produced by IW (Pelinovsky, 1978; Basovich, 1979; Ermakov et al., 1980).

\section{CONCLUSION}

Of course, the different mechanisms of IW-turbulence interaction considered above may act in the ocean simultaneously. However, their relative role depends on the concrete physical condition including the IW wavelength range. Thus, short IW $(\lambda<100 \mathrm{~m})$ dissipate due to turbulent viscosity, the decrement being of the order of $10^{-4} \mathrm{~s}^{-1}$. Longer IW dissipate mainly due to the action of turbulent diffusion, with the decrement weakly dependent on the wave number up to frequencies of the order of the inertial frequency. The total energy dissipation due to this mechanism for the Garrett-Munk spectrum may be estimated as $10^{-4} \mathrm{erg} \mathrm{cm}^{-3} \mathrm{~s}^{-1}$ (a). The corresponding dissipation time, $\tau_{\text {dis }}$, is of the order of 20 days.

At the same time, alternating shear flows in IW may sustain turbulence in the ocean. The characteristic time of turbulent energy growth is of the order of $10^{4} \mathrm{~s}$. The estimation by the Garrett-Munk spectrum gives the value $10^{-5}$ erg $\mathrm{cm}^{-3} \mathrm{~s}^{-1}$ (b) for the flux of energy from IW to turbulence due to this mechanism. The estimates (a) and (b) are different because the first value, in addition to turbulence amplification, includes energy consumption for density deformation.

Comparative estimations of the effectiveness of generation and dissipation of deep-water IW (see the review by Müller and McComas, 1981) indicate a mismatch between characteristic time scales, $\tau_{\text {gen }}$ and $\tau_{\text {dis }}$, of these two processes. Dissipation mechanisms considered by Müller and McComas (1981) (bottom friction, wave breaking, and absorption in critical layers) give a value of $\tau_{\mathrm{dis}}$ of about 100 days, which exceeds considerably the time of generation and non-linear spectrum transformation ( $\tau_{\text {dis }} \sim 20$ days). The mechanism of energy exchange between turbulence and IW without breaking of the latter, as considered here, turns out to be more effective $\left(\tau_{\mathrm{dis}} \sim 20\right.$ days). These losses seem to be sufficient to balance the action of energy sources.

Such an effect seems to be even more essential for low-mode IW in an upper oceanic layer where the influence of intensive sources of IW energy (wind, and surface waves) is considerable (see Roth et al., 1981), while the non-linear interactions of IW are comparatively weak. It means that for upper-layer IW the hypothesis of local energy balance (Ostrovsky and 
Soustova, 1979) seems to be reasonable: IW energy dissipates in the same spectral interval where it is generated by external sources (while the non-linear energy transform along the spectrum is not so important). Of course, this hypothesis needs special experimental verification, but the absence of universal spectra of IW in the upper ocean, and their strong dependence on geographic and synoptic conditions (Roth et al., 1981) may serve as an implicit support for it.

\section{REFERENCES}

Barenblatt, G.I., 1982. Similarity, Self-similarity, and Intermediate Asymptotics. 2nd edn., Gidrometeoizdat, $255 \mathrm{pp}$.

Basovich, A.Ya., 1979. Transformation of the surface wave spectrum by the internal wave. Izv. Akad. Nauk SSSR, Fiz. Atmos. Okeana, 15: 655-661.

Eriksen, C.C., 1978. Measurements and models of fine structure, internal gravity waves, and wave breaking in the deep ocean. J. Geophys. Res., 83: 2989-3009.

Ermakov, S.A., Pelinovsky, E.N. and Talipova, T.G., 1980. Influence of surface-active material upon spectral changes in wind ripple produced by internal waves. Izv. Akad. Nauk SSSR, Fiz. Atmos. Okeana, 19: 1068-1076.

Fua, D., Chimonas, G., Einaudi, F. and Zeman, O., 1982. An analysis of wave-turbulence interaction. J. Atmos. Sci., 39: 2450-2463.

Garrett, C. and Munk, W., 1975. Space-time scales of internal waves: a progress report. J. Geophys. Res., 80: 291-297.

Kitaigorodsky, S.A. and Miropolsky, Yu.Z., 1967. On the theory of turbulent mixing in the upper ocean layer. Izv. Akad. Nauk SSSR, Fiz. Atmos. Okeana, 3: 1196-1209.

Le Blond, P.H., 1966. On the damping of internal waves in the deep ocean. J. Fluid Mech., 25: $121-142$.

Monin, A.S. and Yaglom, A.M., 1971. Statistical Fluid Dynamics. MIT Press, Cambridge, MA, $769 \mathrm{pp}$.

Müller, P. and McComas, C.H., 1981. Dynamics and energy balance of oceanic internal waves. Nonl. Prop. Int. Waves Workshop. San Diego, California, February 27-29, 1981, pp. 181-201.

Ostrovsky, L.A. and Soustova, I.A., 1979. The upper mixed layer of the ocean as an energy sink of internal waves. Okeanologiya, 19: 973-981.

Ostrovsky, L.A., Soustova, I.A. and Tsimring, L.Sh., 1981. The internal wave forcing on the small-scale turbulence in the ocean. Preprint IPF AN SSSR, Gorky, 16 pp.

Pelinovsky, E.N., 1978. The linear theory of surface roughness and its variations at a weak wind. Izv. Akad. Nauk SSSR, Fiz. Atmos. Okeana, 14: 1167-1176.

Pelinovsky, E.N. and Soustova, I.A., 1980. Excitation of small-scale turbulence by internal waves in the ocean with fine structure. In: Fine Structure and Synoptic Variability of Seas, Tallin, 136-139.

Phillips, O.M., 1977. The Dynamics of the Upper Ocean. 2nd edn., Cambridge University Press, 336 pp.

Roth, M.W., Briscoe, M.G. and McComas, C.H., 1981. Internal waves in the upper ocean. J. Phys. Oceanogr., 11: 1234-1247.

Townsend, A.A., 1976. The Structure of Turbulent Shear Flow. 2nd edn., Cambridge University Press, 429 pp. 